\title{
Symmetric Groups under multiset perspective
}

\author{
${ }^{1}$ Yohanna Tella, ${ }^{2}$ Simon Daniel \\ ${ }^{1,2}$ Department of Mathematical Sciences Kaduna State University, Kaduna Nigeria.
}

\begin{abstract}
A multiset is a collection of objects in which repetition of elements is significant. In this paper an attempt to define a symmetric group under multiset context is presented and the analogous to Cayley's theorem is derived.
\end{abstract}

Key words: Multiset, group, symmetric group, Cayley's theorem

\section{Introduction}

Classical set theory states that a given element can appear only once in a set, it assumes that all mathematical objects occur without repetition. Thus there is one number four, one field of complex numbers, etc. So, the only possible relations between two mathematical objects are (1) they are equal, or (2) they are different. In Science and in the ordinary life the situation is not at all like this. In the physical world it is observed that there is an enormous repetition. For instance, there are many hydrogen atoms, many water molecules many strands of DNA, etc. Coins of the same denomination and year, electrons or grains of sand appear to us as the same, despite being obviously separate. This leads to three possible relations between any two physical objects; they are different, they are the same but separate, or they are coinciding and identical, but for the sake of definiteness we say that two physical objects are the same, or equal. If they are indistinguishable, but possibly separate, and identical if they physically coincide (identity is a refinement of equality).

Multisets (sometimes also called bags [7]) are set-like structures where an element can appear more than once [8].Thus, a multiset differs from a set in that each element has a multiplicity, which is a natural number indicating (loosely speaking) how many times it is a member of the multiset. One of the most natural and simple example is the multiset of prime factors of a number $n$. Thus, the number 504 has the factorization $504=2^{3} 3^{2} 7^{1}$ which gives the multiset $[2,2,2,3,3,7]$. Another example is the multiset of solutions of an algebraic equation. Everyone learns in secondary school that a quadratic equation has two (complex) solutions, but in some cases they are both the same number. Thus the multiset of solutions of the equation could be $\{-2,5\}$, or it could be $[3,3]$. In the latter case it has a solution of multiplicity 2 .

The word multiset was coined by De Bruijin in a private communication with D. Knuth in 1981, but the first person that actually used multisets was Richard Dedekind in 1898 [9]. A complete survey of the development of multiset theory can be found in [10].

Research on the multiset theory has not yet gained ground and is still in its infant stages. The research carried out so far shows a strong analogy in the behavior of multisets and sets and it is possible to extend some of the main notions and results of sets to that of multisets for instance, the theoretical aspects of multisets by extending the notions of relations, functions, composition and partition has been explored in [4 ] and [6]. In another related development multiset relation, a partially ordered multiset (pomset), chains and antichains of pomsets has been defined and some results related to multisets and pomsets particularly the analogous of Dilworth's theorem and its dual for pomsets obtained in [5]. The concept of topological spaces in the context of multisets has been put forward in [3]. Here, notion of M-topological space and the concept of open multisets are introduced. Furthermore the notions of basis, sub basis, closed sets, closure and interior in topological spaces are extended to M-topological spaces . Continuous multiset functions are defined and related properties, in particular the comparison of discrete topology and discrete M-topology are established. Recently in [1] and [2], the notion of multigroups, submultigroups, abelian multigroups, normal multigroups and factor multigroups has been introduced. In this paper we define a symmetric multigroup and derive the analogous Cayley's theorem . We presents the paper's preliminaries comprising of some basic definitions, notations and derive some results required in the paper in section 2. We establish the composition of bijective multiset functions defined in [8] as a group and use the result in the definition of symmetric multigroup to derive the analogous of Cayley's theorem in section 3 .

\section{Preliminaries}

Definition 2.1 Formally, a multiset ( mset for short) $M$ over a set $\mathrm{S}$ is a cardinal-valued function. That is, $M$ on $\mathrm{S}$ is a map from $\mathrm{S}$ to the set $\square$ of natural numbers denoted $M: \mathrm{S} \rightarrow \square$. For objects $x \in \mathrm{S}, M(x)$ 
denotes the number of times $x$ appears in $M$ also called the multiplicity of $x$ in $M$. The multiplicity of an element in a mset is axiomatized as unique. i.e $\forall x \forall n \forall m(M(x)=n \wedge M(x)=m \rightarrow n=m)$ (see [7]) $M=\left\{k_{1} / x_{1}, k_{2} / x_{2}, \ldots, k_{n} / x_{n}\right\}=\left[x_{1}, x_{2}, \ldots, x_{n}\right]_{k_{1}, k_{2}, \ldots, k_{n}}$ also means that $M$ is an mset with $x_{1}$ appearing $k_{1}$ times, $x_{2}$ appearing $k_{2}$ times and so on. i.e $M\left(x_{1}\right)=k_{1}, M\left(x_{2}\right)=k_{2}, \ldots, M\left(x_{n}\right)=k_{n}$.

We denote the cardinality of a mset $M$ by $|M|$ where $|M|=\sum_{i=1}^{n} k_{i}$. A mset $M$ is finite if and only if its cardinality is finite. It follows that $M(x)>0 \forall x \in M$. However $M(x)=0$ if and only if $x \notin M$. An mset $M \in \mathrm{M}(\mathrm{S})$ such that $M(x)=0 \forall x \in \mathrm{S}$ is called an empty mset. We denote an empty mset $M$ by $\phi$ and a class of all finite msets over a set $\mathrm{S}$ by $\mathrm{M}(\mathrm{S})$ defined:

$\mathrm{M}(\mathrm{S})=\{M \mid M: \mathrm{S} \rightarrow \square$ and $M(x)=0$ for all but finitely many $x \in M\}$. Here, $\mathrm{S}$ is called the generating set or ground or base set for $\mathrm{M}(\mathrm{S})$.

Definition [8] 2.2 Two msets $M, N \in \mathrm{M}(\mathrm{S})$ are said to be equal denoted $M=N$ if and only if $M(x)=N(x)$ for all $x \in \mathrm{S}$.

Definition 2.3 For any mset $M \in \mathrm{M}(\mathrm{S})$, the predicate $\operatorname{set}(M)$ is defined: $\operatorname{set}(M)=\phi \vee \forall x \forall n(M(x)=n \rightarrow n=1)$. Thus, for any set $\mathrm{S}$, $\operatorname{set}(\mathrm{S})$.

Definition [8] 2.4. Let $M, N \in \mathrm{M}(\mathrm{S}) . \quad M$ is a submultiset (submset for short) of $N$, denoted $M \subseteq N$ or $\mathrm{N} \supseteq \mathrm{M}$ if and only if $M(x) \leq N(x) \quad \forall x \in \mathrm{S}$.

For mset terms $M$ and $N$ we denote $M_{N}$ for unique submset of $M$ that contains copies of the element $N$ in $M$ and nothing else. Denoting $[x]_{t}$ for a mset containing exactly $t$ copies of $x$ we define $M_{x}=[x]_{t}$ if and only if $M(x)=t$ and $\left|M_{x}\right|=\left|[x]_{t}\right|=t$.

Theorem [8] 2.1. Two msets $M, N \in \mathrm{M}(\mathrm{S})$ are equal if and only if $M \subseteq N$ and $N \subseteq M$.

Definition [8] 2.5 Let $M \in \mathrm{M}(\mathrm{S})$. Then the root set $M^{*}$ of $M$ is defined:

$M^{*}=\{x \mid x \in \mathrm{S} \wedge M(x)>0\}$.In other words, if $M=\left[x_{1}, x_{2}, \ldots, x_{n}\right]_{k_{1}, k_{2}, \ldots, k_{n}} k_{i}>0, i \in[1, n]$,

we have $M^{*}=\left[x_{1}, x_{2}, \ldots, x_{n}\right]$. Note that $\operatorname{set}\left(M^{*}\right) \wedge x \in M \leftrightarrow x \in M^{*}$ and For any $M \in \mathrm{M}(\mathrm{S})$, $M^{*} \subseteq M$ (see [8] for details).

Definition [8] 2.6. A multiset $\left[x_{1}, x_{2}, \ldots, x_{n}\right]_{k_{1}, k_{2}, k_{3}, \ldots, k_{n}}=M \in \mathrm{M}(S)$ is regular if and only if $k_{1}=k_{2}=k_{3}=\ldots=k_{n}=k$

\section{Multiset functions}

\section{Symmetric Multigroups}

For functions between arbitrary msets it is required that images of indistinguishable elements of the domain must be indistinguishable elements of the range, but images of distinct elements of the domain need not be distinct elements of the range.

A function $f$ from mset $M$ to mset $N$ is rooted in the function $f$ from $M^{*}$ to $N^{*}$.

We think of $M$ as partitioned into submsets $M_{z}$, and $N$ as partitioned into submsets $N_{z^{\prime}}$. Depending upon the nature of the root function $f$ from $M^{*}$ to $N^{*}$ certain submsets $N_{z^{\prime}}$ will be of the form $N_{f(z)}$ where $z \in M$. We think of the function from $M$ to $N$ as acting upon all copies of $z$ in $M_{z}$ and some copies of $f(z)$ in $N_{f(z)}$ (although which copies of $f(z)$ in $N_{f(z)}$ cannot be determined). 
Definition [8] 3.1. Let $M, N \in \mathrm{M}(\mathrm{S})$. A function from $M$ to $N$ is defined to be a function from $M^{*}$ to $N^{*}$. i.e every function between msets is a set of ordered pair sets.

Definition [8] 3.2. Let $M, N \in \mathrm{M}(\mathrm{S})$ such that $M \subseteq N$. The identity mset function $I: M \rightarrow N$ is just the identity function $I: M^{*} \rightarrow N^{*}$. Note that $M \subseteq N \rightarrow M^{*} \subseteq N^{*}$

Definition [4,8] 3.3. Let $M, N, P \in \mathrm{M}(\mathrm{S})$ and $f: M \rightarrow N, g: N \rightarrow P$ be mset functions. The mset composition function $g \circ f: M \rightarrow P$ is just the composition function $g \circ f: M^{*} \rightarrow P^{*}$.

Definition 3.4 Let $M, N \in \mathrm{M}(\mathrm{S})$ such that $f: M \rightarrow N$ and $g: M \rightarrow N$ are mset functions. Then $f=g$ if and only if $f(x)=g(x)$ for all $x \in M^{*}$.

Definition [8] 3.5. Let $M, N \in \mathrm{M}(\mathrm{S})$.

The function $f: M \rightarrow N$ is an injection if and only if

(i) $f: M^{*} \rightarrow N^{*}$ is an injection and

(ii) $\forall z\left(z \in M^{*} \rightarrow\left|M_{z}\right| \leq\left|N_{f(z)}\right|\right)$.

The function $f: M \rightarrow N$ is a surjection if and only if

(i) $f: M^{*} \rightarrow N^{*}$ is a surjection and

(ii) $\forall z\left(z \in M^{*} \rightarrow\left|M_{z}\right| \geq\left|N_{f(z)}\right|\right)$.

The function $f: M \rightarrow N$ is a bijection if and only if

(i) $f: M^{*} \rightarrow N^{*}$ is a bijection and

(ii) $\forall z\left(z \in M^{*} \rightarrow\left|M_{z}\right|=\left|N_{f(z)}\right|\right)$.

I.e. the function $f: M \rightarrow N$ is a bijection if and only if it is both injective and surjective.

Definition 3.6 [4]. Let $M, N \in \mathrm{M}(\mathrm{S})$. The function $f^{-1}: N \rightarrow M$ is an inverse function of the function $f: M \rightarrow N$ if and only if $f: M \rightarrow N$ is bijective.

Theorem 3.1 [4]. Let $M, N, P \in \mathrm{M}(\mathrm{S})$ such that $f: M \rightarrow N, g: N \rightarrow P$ are bijections. Then $g o f$ is a bijection.

Theorem 3.2 [4]. Let $M, N \in \mathrm{M}(\mathrm{S})$ such that $f: M \rightarrow N$ is a bijection. Then $f^{-1}$ is a bijection

\section{Group of bijective multiset functions}

Let $M \in \mathrm{M}(\mathrm{S})$. We denote the set of bijective mset functions $f: M \rightarrow M$ by $M^{=M}$.

Proposition 3.3 For any $M \in \mathrm{M}(\mathrm{S})$, the identity mset function $I: M \rightarrow M$ defined by $I(x)=x \quad \forall x \in M^{*}$ is bijective.

Proof

Since $I(x)=x \quad \forall x \in M^{*}$ we have $I\left(M^{*}\right) \subseteq M^{*}$ and

For all $x \in M^{*}$ we have $x=I(x) \in I\left(M^{*}\right)$. In particular $M^{*} \subseteq I\left(M^{*}\right)$.

Thus $M^{*}=I\left(M^{*}\right)$ and $I: M^{*} \rightarrow M^{*}$ is bijective.

Now for all $x \in M^{*} .\left|M_{x}\right|=\left|M_{I(x)}\right|$. Hence $I: M \rightarrow M$ is bijective.

Proposition 3.4 Let $M \in \mathrm{M}(\mathrm{S})$ and $f \in M^{=M}$. Then $f o I=I o f=f$ where $I \in M^{=M}$ is identity.

Proof.

Let $x \in M^{*}$. We show that $\operatorname{Iof}(x)=f(x)$ and $\operatorname{Iof}(x)=f o I(x)$.

Now $\operatorname{Iof}(x)=I(f(x))=f(x)$. Thus, Iof $=f$.

But $\operatorname{Iof}(x)=f(x) \rightarrow \operatorname{Iof}(x)=f(x)=f((I(x))=f o I(x)$ 
Thus, $f o I=I o f=f$

Proposition 3.5 For any $M \in \mathrm{M}(\mathrm{S}), M^{=M} \neq \phi$.

Proof

The prove follows from proposition 3.3

Proposition 3.6. For any $f \in M^{=M}$, we have

(i) $f^{-1} \in M^{=M}$ (ii) $f o f^{-1}=f^{-1}$ of $=I \in M^{=M}$

Proof:

Let $f \in M^{=M}$. Then (i) follows from theorem 3.2 where $M=N$.

(ii) $f o f^{-1}=f^{-1} o f=I_{M}=I \in M^{=M}$ (see [6] for details) where $M=N$.

Proposition 3.7. For any $f, g \in M^{=M}$, we have $f o g, g o f \in M^{=M}$

Proof

The results follows from theorem 3.1 where $M=N$.

Proposition 3.8. Let $M \in \mathrm{M}(\mathrm{S})$. The pair $\left(M^{=M}, o\right)$ is a group.

Proof:

Let $f, g \in M^{=M}$. It is clear that $g^{-1} \in M^{=M}$ and $f \circ g \in M^{=M}$ (from propositions 3.6 and 3.7).

In particular, $f o g^{-1} \in M^{=M}$. Thus, $\left(M^{=M}, o\right)$ is a group.

III Symmetric multigroups.

Definition [1] 3.7 A multiset $M \in \mathrm{M}(S)$ is called a multigroup if and only if $M^{*}$ is a group.

Definition 3.8 A permutation of a multiset $M \in \mathrm{M}(S)$ is a bijective mapping $\varphi: M \rightarrow M$.

It is clear from proposition 3.8 that the permutations of a multiset forms a group called symmetric multigroup where the composition of bijective multiset functions is its binary operation. We denote the symmetric multigroup of a multiset $M \in \mathrm{M}(S)$ by $S_{m u l}(M)$

A subgroup of $S_{m u l}(M)$ is called a permutation multigroup.

Example $\quad$ 3.1 Let $S=\{e, a, b, c\} \quad$ and $\quad\{e, e, e, a, a, b, b, b, c, c\}=M \in \mathrm{M}(S)$.

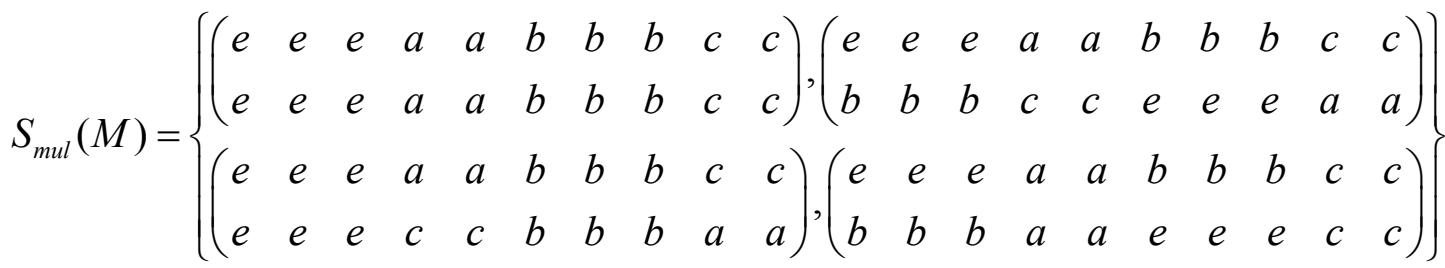

$=\left\{\left(\left(\begin{array}{l}e \\ e\end{array}\right)_{3}\left(\begin{array}{l}a \\ a\end{array}\right)_{2}\left(\begin{array}{l}b \\ b\end{array}\right)_{3}\left(\begin{array}{l}c \\ c\end{array}\right)_{2}\right),\left(\left(\begin{array}{l}e \\ b\end{array}\right)_{3}\left(\begin{array}{l}a \\ c\end{array}\right)_{2}\left(\begin{array}{l}b \\ e\end{array}\right)_{3}\left(\begin{array}{l}c \\ a\end{array}\right)_{2}\right),\left(\left(\begin{array}{l}e \\ e\end{array}\right)_{3}\left(\begin{array}{l}a \\ c\end{array}\right)_{2}\left(\begin{array}{l}b \\ b\end{array}\right)_{3}\left(\begin{array}{l}c \\ a\end{array}\right)_{2}\right),\left(\left(\begin{array}{l}e \\ b\end{array}\right)_{3}\left(\begin{array}{l}a \\ a\end{array}\right)_{2}\left(\begin{array}{l}b \\ e\end{array}\right)_{3}\left(\begin{array}{l}c \\ c\end{array}\right)_{2}\right)\right\}$

Notes that the order $\left|S_{m u l}(M)\right|=4 \neq 10$ !

However, if the multiset $M \in \mathrm{M}(S)$ is regular, where $M=\left[x_{1}, x_{2}, \ldots, x_{n}\right]_{k_{1}, k_{2}, k_{3}, \ldots, k_{n}}$ and $k_{1}=k_{2}=k_{3}=\ldots=k_{n}$ then $\left|S_{m u l}(M)\right|=n ! \neq\left(\sum_{i=1}^{n} k_{i}\right) ! \quad k_{i}>0$ and $n=\left|M^{*}\right|$

Example 3.2 Let $S=\{e, a, b\}$ and $\{e, e, e, a, a, a, b, b, b\}=M \in \mathrm{M}(S)$. 


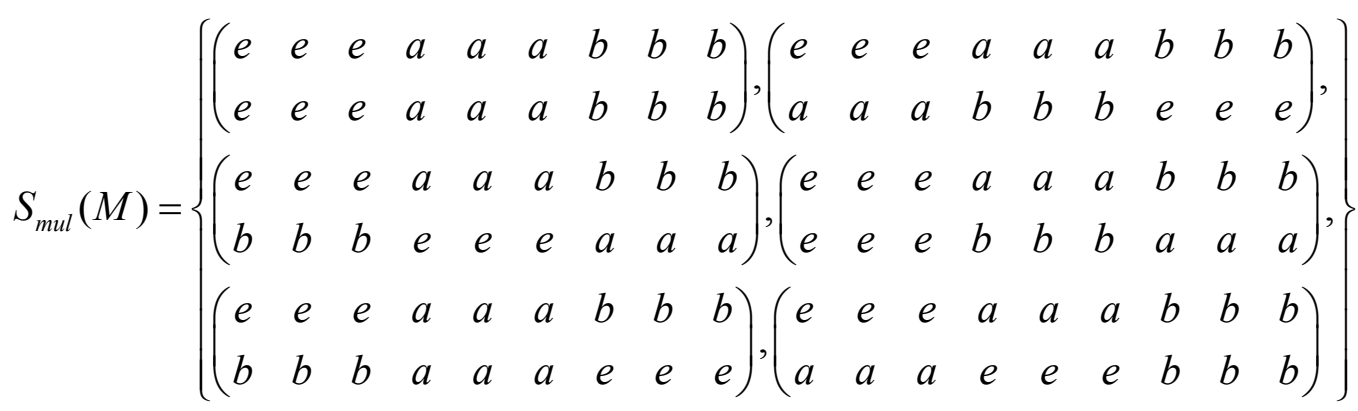

i.e $S_{m u l}(M)=\left\{\begin{array}{l}\left(\left(\begin{array}{l}e \\ e\end{array}\right)_{3}\left(\begin{array}{l}a \\ a\end{array}\right)_{3}\left(\begin{array}{l}b \\ b\end{array}\right)_{3}\right),\left(\left(\begin{array}{l}e \\ a\end{array}\right)_{3}\left(\begin{array}{l}a \\ b\end{array}\right)_{3}\left(\begin{array}{l}b \\ e\end{array}\right)_{3}\right),\left(\left(\begin{array}{l}e \\ b\end{array}\right)_{3}\left(\begin{array}{l}a \\ e\end{array}\right)_{3}\left(\begin{array}{l}b \\ a\end{array}\right)_{3}\right), \\ \left(\left(\begin{array}{l}e \\ e\end{array}\right)_{3}\left(\begin{array}{l}a \\ b\end{array}\right)_{3}\left(\begin{array}{l}b \\ a\end{array}\right)_{3}\right),\left(\left(\begin{array}{l}e \\ b\end{array}\right)_{3}\left(\begin{array}{l}a \\ a\end{array}\right)_{3}\left(\begin{array}{l}b \\ e\end{array}\right)_{3}\right),\left(\left(\begin{array}{l}e \\ a\end{array}\right)_{3}\left(\begin{array}{l}a \\ e\end{array}\right)_{3}\left(\begin{array}{l}b \\ b\end{array}\right)_{3}\right)\end{array}\right\}$

$\left|S_{m u l}(M)\right|=\left|M^{*}\right| !=3 !=3 \times 2 \times 1=6$.

Notes that if the multiset $M=\left[x_{1}, x_{2}, \ldots, x_{n}\right]_{k_{1}, k_{2}, k_{3}, \ldots, k_{n}}$ is such that $k_{i} \neq k_{j}$ for $i \neq j$, then

$S_{m u l}(M)$ is trivial. That is $S_{m u l}(M)=\{I\}$ where $I=\left(\left(\begin{array}{l}x_{1} \\ x_{1}\end{array}\right)_{k_{1}}\left(\begin{array}{l}x_{2} \\ x_{2}\end{array}\right)_{k_{2}}\left(\begin{array}{l}x_{3} \\ x_{3}\end{array}\right)_{k_{3}} \ldots\left(\begin{array}{l}x_{n} \\ x_{n}\end{array}\right)_{k_{n}}\right)$

Proposition 3.9. For any regular multigroup $\mathrm{G} \in \mathrm{M}(S), G^{*}$ is isomorphic to a subgroup of $S_{m u l}(G)$

Proof:

Let $\lambda_{g}: G^{*} \rightarrow G^{*}$ be a mapping for a fixed $g \in G^{*}$ defined:

$\lambda_{g}(x)=g x$ for all $x \in G^{*}$.

This is a well-defined mapping. Indeed if $x=y$ then $g x=g y$ so that $\lambda_{g}(x)=\lambda_{g}(y)$.

Next we show that $\lambda_{g}$ is one-to-one. To see this, suppose that $\lambda_{g}(x)=\lambda_{g}(y)$. Then $g x=g y$ and by cancellation property, $x=y$. To see that $\lambda_{g}$ is onto, let $y \in G^{*}$. Then $g^{-1} y \in G^{*}$ and $\lambda_{g}\left(g^{-1} y\right)=y$. Hence, $\lambda_{g}: G^{*} \rightarrow G^{*}$ is a bijection

Since $\mathrm{G} \in \mathrm{M}(S)$ is regular, we have $\left|G_{x}\right|=\left|G_{\lambda_{g}(x)}\right|$.

Hence, $\lambda_{g}: G \rightarrow G$ is a bijection. In particular $\lambda_{g} \in S_{m u l}(M)$.

Next, we define $\Lambda: G^{*} \rightarrow S_{m u l}(G)$ by $\Lambda(g)=\lambda_{g}$. This is a well-defined mapping. For if $g_{1}=g_{2}$ then $g_{1} x=g_{2} x$ for all $x \in G^{*}, \quad$ that $\quad$ is, $\quad \lambda_{g_{1}}(x)=\lambda_{g_{2}}(x)$ for all $x \in G^{*} \quad$ and hence $\quad \lambda_{g_{1}}=\lambda_{g_{2}}$, i.e $\Lambda\left(g_{1}\right)=\Lambda\left(g_{2}\right)$. Now given $g_{1}, g_{2} \in G^{*}$ we have $\lambda_{g_{1} g_{2}}(x)=\left(g_{1} g_{2}\right) x=g_{1}\left(g_{2} x\right)=\lambda_{g_{1}}\left(g_{2} x\right)=\lambda_{g_{1}} \lambda_{g_{2}}(x)$ for all $x \in G^{*}$

Thus, $\Lambda\left(g_{1} g_{2}\right)=\lambda_{g_{1} g_{2}}=\lambda_{g_{1}} \lambda_{g_{2}}=\Lambda\left(g_{1}\right) \Lambda\left(g_{2}\right)$, and so $\Lambda$ is a homomorphism.

Finally we show that $\Lambda$ is one-to-one. Indeed, if $\Lambda\left(g_{1}\right)=\Lambda\left(g_{2}\right)$ then $\lambda_{g_{1}}(x)=\lambda_{g_{2}}(x)$ for all $x \in G^{*}$. In particular, $\lambda_{g_{1}}(e)=\lambda_{g_{2}}(e)$. That is, $g_{1} e=g_{2} e$ or $g_{1}=g_{2}$

Now for onto, let $\sigma \in S_{m u l}(G)$. Clearly for some $g \in G^{*}$ we have $\sigma=\lambda_{g}=\Lambda(g)$.

Thus, for all $\sigma \in S_{m u l}(G)$, there exist $g \in G^{*}$ such that $\sigma=\Lambda(g)$.

Hence, $G^{*} \cong S_{m u l}(G)$

Note that this does not hold for any irregular multigroup. 


\section{References}

[1]. Y. Tella and S. Daniel; The Study of Group Theory in the context of Multiset Theory, International journal of Science and Technology, 8(2) (2013)

[2]. SK Nazamul, P. Majumdar, and S.K. Samanta, On Multisets and Multigroups, Anals of Fuzzy Mathematics and Informatics (2013)

[3]. K. P. Girisha,_, Sunil Jacob John, On Multiset Topologies, Theory and Applications of Mathematics \& Computer Science 2 (1) (2012).

[4]. Y.Tella and S.Daniel; Functions in Multiset Context International Journal of Physical science, 4(2). 2010

[5]. K.P. Girish and Sunil Jacob John, General Relations between partially ordered multisets and their chains and antichains, Math. Commun., 14 (2) (2009).

[6]. K.P. Girish and Sunil Jacob John, Relations and Functions in Multiset Context, Information Sciences 179 (2009).

[7]. R.R. Yager, On the theory of bags, International Journal of General System 13 (1986).

[8]. W. D. Blizard, Multiset Theory, Notre Dame Journal of Logic 30 (1989).

[9]. W. D. Blizard, Dedeking Multisets and Functions shells, Theoret. Comput. Sci. 110 (1993)

[10]. W. D. Blizard, The development of Multiset Theory, Modern Logic (1991) 